\title{
Renouvellement des chaires depuis 1945
}

\section{(2) OpenEdition \\ 1 Journals}

Édition électronique

URL : https://journals.openedition.org/annuaire-cdf/16538

DOI : $10.4000 /$ annuaire-cdf. 16538

ISBN : 978-2-7226-0572-5

ISSN : 2109-9227

Éditeur

Collège de France

Édition imprimée

Date de publication : 30 décembre 2020

Pagination : 697-718

ISBN : 978-2-7226-0516-9

ISSN : 0069-5580

\section{Référence électronique}

"Renouvellement des chaires depuis 1945 », L'annuaire du Collège de France [En ligne], 118 | 2020, mis en ligne le 04 mai 2021, consulté le 22 août 2022. URL : http://journals.openedition.org/annuaire-cdf/ 16538 ; DOI : https://doi.org/10.4000/annuaire-cdf.16538 


\section{RENOUVELLEMENT DES CHAIRES DEPUIS 1945}

\section{De 1945 à $2014^{1}$}

\section{Ancienne chaire}

Nouvelle chaire

\section{$1945^{2}$}

Langue et littérature chinoises Henri MASPERO (1921-1945)

Langue et littérature arabes William MARÇAIS (1927-1943)

Histoire ancienne de l'Orient sémitique Isidore LÉVY (1932-1941)

Physique mathématique (chaire créée pour Albert EINSTEIN en 1933)
Langue et littérature chinoises Paul DeMiÉviLle (1946-1964)

Histoire du monde arabe Jean SAUVAGET (1946-1950)

Philologie et archéologie assyrobabyloniennes

Édouard DHORME (1945-1951)

Physique atomique et moléculaire Francis PERRIN (1946-1972)

1. Pour un historique des chaires depuis 1800 , on pourra se reporter aux volumes antérieurs de l'Annuaire. Voir par exemple Annuaire du Collège de France 2014-2015. Résumé des cours et travaux, $115^{e}$ année, Paris, Collège de France, 2016, p. 37-70.

2. L'année indiquée est celle de la délibération de l'Assemblée des professeurs sur la création, le maintien ou la transformation de la chaire. 
Poétique

Paul VALÉRY (1937-1945)

Psychologie collective

Maurice HALBWACHS (1944-1945)

Physique générale et expérimentale

Paul LANGEVIN (1909-1946)

Langues, histoire et archéologie de l'Asie centrale

Paul PELLIOT (1911-1945)

Histoire naturelle des corps organisés André MAYER (1922-1946)

Histoire et philologie indochinoises Jean PRZYLUSKI (1931-1944)

Civilisation américaine

Bernard FAŸ (révoqué) (1932-1945)

Géographie économique et politique André SIEGFRIED (1933-1946)

Histoire du vocabulaire français Mario RoQUES (1937-1946)

Création

Henri BREUIL (1929-1947)

Égyptologie

Pierre LACAU (1938-1947)

Histoire de la colonisation

Edmond CHASSIGNEUX (1939-1946)

Biologie générale

Jacques DUCLAUX (1931-1948)
Histoire des créations littéraires en France Jean POMMIER (1946-1964)

Histoire de la civilisation italienne Augustin RENAUDET (1946-1950)

\section{6}

Théorie des équations différentielles et fonctionnelles

Jean LERAY (1947-1978)

Archéologie paléochrétienne et byzantine André GRABAR (1946-1966)

Biochimie générale et comparée Jean RoCHE (1947-1972)

Histoire et philologie indochinoises Émile GASPARDONE (1946-1965)

Histoire de la civilisation de l'Amérique du Nord

Marcel GIRAUD (1947-1971)

Étude du monde tropical

Pierre GOUROU (1947-1970)

Langue et littérature françaises du Moyen Âge

Félix LECOY (1947-1974)

Civilisations de l'Extrême-Orient

Paul Mus (1946-1969)

\section{7}

Géographie historique de la France Roger DiON (1948-1968)

Égyptologie

Pierre MONTET (1948-1956)

Histoire de l'expansion de l'Occident Robert MONTAGNÉ (1948-1954)

Neurophysiologie générale Alfred FESSARD (1949-1971) 
Langues et littératures d'origine germanique

Ernest TONNELAT (1934-1948)

Antiquités nationales

Albert GRENIER (1936-1948)
Langues et littératures d'origine germanique

Fernand MossÉ (1949-1956)

Civilisation indo-européenne

Georges DuMÉZIL (1949-1968)
Physique théorique

Léon BRILLOUIN (1932-1949)

Histoire de la civilisation moderne

Lucien FEBVRE (1933-1949)

Psychologie et éducation de l'enfance

Henri WALLON (1937-1949)

\section{Médecine}

René LERICHE (1937-1950)

Histoire de la civilisation italienne

Augustin RENAUdET (1946-1950)

Histoire du monde arabe

Jean SAUVAGET (1946-1950)

Création (fondation de la ville de Paris)
Physique théorique

Jean LAVAL (1950-1970)

Histoire de la civilisation moderne

Fernand BRAUDEL (1950-1972)

Psychologie des arts plastiques

René HuYGHE (1951-1976)

1950

Médecine expérimentale

Antoine LACASSAGNE (1951-1954)

Littérature et civilisation italiennes

André PÉZARD (1951-1963)

Langue et littérature arabes

Gaston WIET (1951-1959)

Histoire et structure sociales de Paris et de la région parisienne

Louis CHEVALIER (1952-1981)

1951

Langues et littératures slaves

André MAZON (1923-1951)

Physiologie des sensations

Henri PIÉRON (1923-1951)

Histoire de la philosophie au Moyen Âge Étienne GILSON (1932-1950)

Histoire des religions

Jean BARUZI (1933-1951)

Langue et littérature sanscrites

Jules BLOCH (1937-1951)
Langues et littératures slaves

André VAILLANT (1952-1962)

Physique mathématique

André LICHNEROWICZ (1952-1986)

Histoire et technologie des systèmes philosophiques

Martial GUÉROULT (1951-1962)

Histoire des religions

Henri-Charles PUECH (1952-1972)

Langues et littératures de l'Inde Jean FILLIOZAT (1952-1978) 
Philosophie

Louis LAVELLE (1941-1951)

Histoire de la langue latine

Alfred ERNOUT (1944-1951)

Philologie et archéologie assyrobabyloniennes

Édouard DHORME (1945-1951)

Radiobiologie expérimentale

Antoine LACASSAGNE (nommé en 1951, titulaire de la chaire de Médecine expérimentale créée l'année précédente)
Philosophie

Maurice MERLEAU-PONTY (1952-1961)

Littérature latine

Pierre COURCELLE (1952-1980)

Assyriologie

René LABAT (1952-1974)

Histophysiologie

Jacques BENOIT (1952-1966)
Histoire des arts de l'Orient musulman Albert GABRIEL (1941-1953)

Littérature latine du Moyen Âge Edmond FARAL (1925-1954)

Embryogénie comparée Emmanuel FAURÉ-FrÉMIET (1928-1954)

Civilisation romaine

André PIGANIOL (1942-1954)

Médecine expérimentale

Antoine LACASSAGNE (1951-1954)
Archéologie de l'Asie occidentale Claude SCHAEFFER-FORRER (1954-1969)

1954

Analyse des faits économiques et sociaux François PERROUX (1955-1974)

Embryologie expérimentale

Étienne WOLFF (1955-1974)

Civilisation romaine

Jean GAGÉ (1955-1972)

Médecine expérimentale

Charles OBERLING (1955-1960)
Sociologie et sociographie musulmanes Louis MASSIGNON (1926-1954)

Aérolocomotion mécanique et biologique Étienne OEHMICHEN (1939-1955)

Chimie organique

Charles DUFRAISSE (1942-1955)

Histoire de l'expansion de l'Occident Robert MONTAGNÉ (1948-1954)
Sociologie musulmane

Henri LAOUST (1956-1975)

Algèbre et géométrie

Jean-Pierre SERRE (1956-1994)

Chimie organique des hormones

Alain HoREAU (1956-1980)

Histoire sociale de l'Islam contemporain Jacques BERQUE (1956-1981)

\section{6}

\section{Égyptologie}

Pierre MONTET (1948-1956)
Philologie et archéologie égyptiennes Étienne DRIOTON (1957-1960) 
Langues et littératures d'origine germanique

Fernand Mossé (1949-1956)

Histoire du travail

Émile COORNAERT (1936-1957)

Chimie nucléaire

Frédéric JOLIOT (1937-1958)

Création

Gaston WIET (1951-1959)

méditerranéenne

Paul FALLOT (1938-1960)

Médecine expérimentale

Charles OBERLING (1955-1960)

Philologie et archéologie égyptiennes Étienne DRIOTON (1957-1960)

Philosophie

Maurice MERLEAU-PONTY (1952-1961)

\section{2}

Histoire et technologie des systèmes philosophiques

Martial GUÉROULT (1951-1962)

Langues et littératures slaves

André VAILLANT (1952-1962)
Langues et littératures d'origine germanique

Robert MINDER (1957-1973)

1958

Démographie sociale : la vie des populations

Alfred SAUVY (1959-1969)

Physique nucléaire

Louis LEPRINCE-RINGUET (1959-1972)

Anthropologie sociale

Claude LÉVI-STRAUSS (1959-1982)

1959

Magnétisme nucléaire

Anatole ABRAGAM (1960-1985)

1960

Méthodes physiques de l'astronomie André LALLEMAND (1961-1974)

Médecine expérimentale

Bernard HALPERN (1961-1975)

Philologie et archéologie égyptiennes

Georges POSENER (1961-1978)

1961

Philosophie de la connaissance Jules VuILLEMIN (1962-1990)

Histoire de la pensée philosophique Jean HyPPOLITE (1963-1968)

Hébreu et araméen

André DUPONT-SOMMER (1963-1971) 
Physique cosmique

Alexandre DAUVILLIER (1944-1962)

Langue et littérature chinoises

Paul DÉMIÉVILLE (1946-1964)

Histoire des créations littéraires en France Jean POMMIER (1946-1964)

Littérature et civilisation italiennes André PÉZARD (1951-1963)

Création

Création

Langues et littératures de la péninsule ibérique et de l'Amérique latine

Marcel BATAILlON (1945-1965)

Histoire et philologie indochinoises Émile GASPARDONE (1946-1965)

Morphologie expérimentale et endocrinologie

Robert COURRIER (1938-1966)

Archéologie paléochrétienne et byzantine André GRABAR (1946-1966)

Histophysiologie

Jacques BENOIT (1952-1966)

Georges DuMÉZIL (1949-1968)
Astrophysique théorique

Jean-Claude PECKER (1964-1988)

Histoire et civilisations de l'Asie centrale

Louis HAMBIS (1965-1977)

Littérature française moderne

Georges BLIN (1965-1988)

Archéologie et histoire de la Gaule

Paul-Marie DUVAL (1964-1982)

Physique théorique des particules élémentaire

Jacques PRENTKI (1965-1983)

Génétique cellulaire

François JACOB (1965-1991)

\section{5}

Langues et littératures de la péninsule ibérique et de l'Amérique latine

Israël RÉVAH (1966-1973)

Étude du monde chinois : institutions et concepts

Rolf A. STEIN (1966-1981)

Physiologie cellulaire

François MOREL (1967-1993)

Histoire et civilisation de Byzance

Paul LEMERLE (1967-1973)

Biologie moléculaire

Jacques MONOD (1967-1973)

1968

Préhistoire

André LEROI-GOURHAN (1969-1982)

\section{9}

Géographie historique de la France

Géographie du continent européen

Maurice LE LANNOU (1969-1976) 
Archéologie de l'Asie occidentale

Claude SCHAEFFER-FORRER (1954-1969)

Démographie sociale : la vie des populations

Alfred SAUVY (1959-1969)

Histoire de la pensée philosophique Jean HyPPOLITE (1963-1968)
Histoire des sociétés médiévales

Georges DuBY (1970-1991)

Sociologie de la civilisation moderne Raymond ARON (1970-1978)

Histoire des systèmes de pensée Michel FouCAULT (1970-1984)
Civilisations de l'Extrême-Orient

Paul Mus (1946-1969)

Étude du monde tropical

Pierre Gourou (1947-1970)

Physique théorique

Jean LAVAL (1950-1970)

Histoire de la civilisation de l'Amérique du Nord

Marcel GIRAUD (1947-1971)

Neurophysiologie générale

Alfred FESSARD (1949-1971)

Hébreu et araméen

André DUPONT-SOMMER (1963-1971)

Francis PERRIN (1946-1972)

Biochimie générale et comparée

Jean RocHE (1947-1972)

Physique nucléaire

Louis LEPRINCE-RINGUET (1959-1972)

Grammaire comparée

Émile BENVENISTE (1937-1972)
Art et civilisation de la Renaissance en Italie

André CHASTEL (1970-1984)

Étude du bouddhisme

André BAREAU (1971-1991)

Physique de la matière condensée

Pierre-Gilles DE GENNES (1971-2004)

\section{1}

Anthropologie physique

Jacques RUFFIÉ (1972-1992)

Neurophysiologie

Yves LAPORTE (1972-1991)

Hébreu et araméen

André CAQUOT (1972-1994)

1972

Physique atomique et moléculaire

Claude COHEN-TANNOUDJI (1973-2004)

Biochimie cellulaire

François GROS (1973-1996)

Physique corpusculaire

Marcel FROISSART (1973-2004)

Langues et civilisation de l'Asie Mineure

Emmanuel LAROCHE (1973-1985) 
Mathématique et mécanique

Szolem MANDELBROJT (1938-1972)

Histoire de la civilisation moderne

Fernand BRAUDEL (1950-1972)

Histoire des religions

Henri-Charles PUECH (1952-1972)

Civilisation romaine

Jean GAGÉ (1955-1972)

Langues et littératures d'origine germanique

Robert MINDER (1957-1973)

Langues et littératures de la péninsule ibérique et de l'Amérique latine

Israël RÉVAH (1966-1973)

Biologie moléculaire

Jacques MONOD (1967-1973)

Histoire et civilisation de Byzance

Paul LEMERLE (1967-1973)

Étienne WOLFF (1955-1974)

Méthodes physiques de l'astronomie

André LALLEMAND (1961-1974)

Louis ROBERT (1939-1974)

Langue et littérature françaises du Moyen Âge

Félix LECOY (1947-1974)

Assyriologie

René LABAT (1952-1974)

Analyse des faits économiques et sociaux François PERROUX (1955-1974)

Sociologie musulmane

Henri LAOUST (1956-1975)
Analyse mathématique des systèmes et de leur contrôle

Jacques-Louis LIONS (1973-1998)

Histoire de la civilisation moderne

Emmanuel LE RoY LADURIE (1973-1999)

La Grèce et la formation de la pensée morale et politique

Jacqueline DE ROMILLY (1973-1984)

Théorie des groupes

Jacques TITS (1973-2000)

Étude comparée des religions antiques

Jean-Pierre VERNANT (1975-1984)

Histoire sociale et intellectuelle de la Chine Jacques GERNET (1975-1992)

Physiologie du développement

Alfred JosT (1974-1987)

Histoire des mentalités religieuses dans l'Occident moderne

Jean DELUMEAU (1975-1994)

\section{4}

Communications cellulaires

Jean-Pierre CHANGEUX (1975-2006)

Neuropsychologie du développement Julian DE AJURIAGUERRA (1975-1981)

Sémiologie littéraire

Roland BARTHES (1976-1980)

Invention, technique et langage en musique Pierre BoulEZ (1976-1995)

Histoire de Rome

Paul-Marie VEYNE (1976-1999)

Histoire et civilisation du monde byzantin Gilbert DAGRON (1975-2001)

Langue et littérature arabes classiques André MiQuEL (1976-1997) 
Psychologie des arts plastiques René HuYGHE (1951-1976)

Médecine expérimentale Bernard HALPERN (1961-1975)

Géographie du continent européen Maurice LE LANNOU (1969-1976)

Histoire et civilisations de l'Asie centrale Louis HAMBIS (1965-1977)

Philologie et archéologie égyptiennes Georges POSENER (1961-1978)

Théorie des équations différentielles et fonctionnelles

Jean LERAY (1947-1978)

Langues et littératures de l'Inde

Jean FILLIOZAT (1952-1978)

Sociologie de la civilisation moderne

Raymond ARON (1970-1978)

Chimie organique des hormones

Alain HOREAU (1956-1980)

Sémiologie littéraire

Roland BARTHES (1976-1980)

Littérature latine

Pierre COURCELLE (1952-1980)
Histoire de la création artistique en France Jacques THUILLIER (1977-1998)

Médecine expérimentale

Jean DAUSSET (1977-1987)

Christianisme et gnoses dans l'Orient préislamique

Antoine GuILLAUMONT (1977-1986)

1977

Sociographie de l'Asie du Sud-Est

Lucien BERNOT (1978-1985)

1978

Égyptologie

Jean LECLANT (1979-1990)

1979

Droit international

René-Jean DuPUY (1979-1989)

Civilisation japonaise

Bernard FRANK (1979-1996)

Chimie des interactions moléculaires

Jean-Marie LEHN (1979-2010)

1980

Bioénergétique cellulaire

Pierre JOLIOT (1981-2002)

Études comparées de la fonction poétique

Yves BONNEFOY (1981-1993)

1981

Sociologie

Pierre BOURDIEU (1981-2001) 
Histoire et structure sociales de Paris et de la région parisienne

Louis CHEVALIER (1952-1981)

Étude du monde chinois : institutions et concepts

Rolf A. STEIN (1966-1981)
Étude comparée des sociétés africaines Françoise HÉRITIER (1982-1998)

Histoire de la pensée hellénistique et romaine

Pierre HADOT (1982-1991)
Histoire sociale de l'Islam contemporain

Jacques BERQUE (1956-1981)

Préhistoire

André LEROI-GOURHAN (1969-1982)

Neuropsychologie du développement Julian DE AJURIAGUERRA (1975-1981)

Claude LÉVI-STRAUSS (1959-1982)

Archéologie et histoire de la Gaule Paul-Marie DuvAL (1964-1982)

Physique théorique des particules élémentaires

Jacques PRENTKI (1964-1983)
Physique statistique

Philippe NOZIÈRES (1983-2001)

Paléoanthropologie et préhistoire Yves COPPENS (1983-2005)

Neuropharmacologie Jacques GLOWINSKI (1982-2006)

\section{3}

Histoire du monde indien

Gérard FUSSMAN (1984-2011)

Antiquités nationales

Christian GoudinEAU (1984-2010)

Analyse et géométrie

Alain CONNES (1984-2017)

\section{4}

La Grèce et la formation de la pensée morale et politique

Jacqueline DE ROMILLY (1973-1984)

Étude comparée des religions antiques Jean-Pierre VERNANT (1975-1984)

\section{5}

Histoire des systèmes de pensée Michel FouCAULT (1970-1984)

Art et civilisation de la Renaissance en Italie

André CHASTEL (1970-1984)

Langues et civilisation de l'Asie Mineure Emmanuel LAROCHE (1973-1985)
Tradition et critique des textes grecs Jean IRIGOIN (1986-1992)

Grammaire et pensée allemandes Jean-Marie ZEMB (1986-1998)

Épistémologie comparative Gilles-Gaston GRANGER (1986-1991)

Histoire de la France contemporaine Maurice AGULHON (1986-1997)

Assyriologie

Paul GARELLI (1986-1995) 
Physique mathématique

André LiCHNEROWICZ (1952-1986)

Magnétisme nucléaire

Anatole ABRAGAM (1960-1985)

Christianisme et gnoses dans l'Orient préislamique

Antoine GuILLAUMONT (1977-1986)

Sociographie de l'Asie du Sud-Est Lucien BERNOT (1978-1985)

Jean DAUSSET (1977-1987)

Physiologie du développement

Alfred Jost (1974-1987)

Astrophysique théorique

Jean-Claude PECKER (1964-1988)

Droit international

René-Jean DuPuY (1979-1989)

Création

\author{
Analyse économique \\ Edmond MALINVAUD (1987-1993) \\ Géodynamique \\ Xavier LE PICHON (1986-2008) \\ Théorie linguistique \\ Claude HAGÈGE (1988-2006) \\ Rhétorique et société en Europe \\ (XVI $-X V I I^{e}$ siècles) \\ Marc FuMAROLI (1987-2002)
}

\section{7}

Embryologie cellulaire et moléculaire Nicole LE DOUARIN (1988-2000)

\section{8}

Médecine expérimentale

Pierre CORVOL (1989-2012)

\section{9}

Astrophysique observationnelle

Antoine LABEYRIE (1991-2014)

Histoire des syncrétismes de la fin de l'Antiquité

Michel TARDIEU (1991-2008)

Chaire européenne

Harald WEINRICH (1989-1990)

1990

Littérature française moderne

Histoire de la Chine moderne

Pierre-Étienne WILL (1991-2014)

Égyptologie

Jean YoYOTTE (1991-1997)

1991

Philosophie de la connaissance Jules VuILLEMIN (1962-1990)
Langues et littératures romanes Harald WEINRICH (1992-1998) 
Histoire des sociétés médiévales

Georges DUBY (1970-1991)

Neurophysiologie

Yves LAPORTE (1972-1991)

Épistémologie comparative

Gilles-Gaston GRANGER (1986-1990)

Chaire européenne
Histoire de l'Occident méditerranéen au Moyen Âge

Pierre TOUBERT (1992-2003)

Physiologie de la perception et de l'action Alain BERTHOZ (1992-2010)

Histoire et anthropologie des sociétés mésoet sud-américaines

Nathan WACHTEL (1992-2005)

Wolf LEPENIES (1991-1992)

\section{2}

Génétique cellulaire

François JACOB (1964-1991)

Étude du bouddhisme

André BAREAU (1971-1991)
Génétique moléculaire

Pierre CHAMBON (1993-2002)

Histoire moderne et contemporaine du monde russe

François-Xavier CoQUIN (1993-2001)

Histoire sociale et intellectuelle de la Chine Langues et religions indo-iraniennes Jacques GERNET (1975-1992)

Histoire de la pensée hellénistique et romaine

Pierre HADOT (1982-1991)

Chaire européenne

Création

1993

Anthropologie physique

Jacques RUFFIÉ (1972-1992)

Études comparées de la fonction poétique Yves BONNEFOY (1981-1993)

Tradition et critique des textes grecs Jean IRIGOIN (1986-1992)

Chaire européenne

Chaire internationale
Jean KELLENS (1993-2014)

Histoire économique et monétaire de l'Orient hellénistique

Georges LE RIDER (1993-1998)

Umberto ECO (1992-1993)

Chaire internationale

Bronislaw GEREMEK (1992-1993)
Fondements et principes de la reproduction humaine

Étienne BAULIEU (1993-1998)

Littératures de la France médiévale Michel ZINK (1994-2016)

Les civilisations de l'Europe au néolithique et à l'âge du bronze

Jean GUILAINE (1994-2007)

Werner HILDENBRAND (1993-1994)

Guangda ZHANG (1993-1994) 
Physiologie cellulaire

François MOREL (1967-1993)

Hébreu et araméen

André CAQUOT (1972-1994)

Histoire des mentalités religieuses dans l'Occident moderne

Jean DELUMEAU (1975-1994)

Analyse économique

Edmond MALINVAUD (1987-1993)

Chaire européenne

Chaire internationale

Algèbre et géométrie

Jean-Pierre SERRE (1956-1994)

Invention, technique et langage en musique Pierre BoulEZ (1976-1995)

Assyriologie

Paul GARELLI (1986-1995)

Chaire européenne

Chaire internationale

Biochimie cellulaire

François GROS (1973-1996)

Chaire européenne

Chaire internationale

ivilisation japonaise

Bernard FRANK (1979-1996)

Égyptologie

Jean YOYOTTE (1991-1997)
Biologie moléculaire des plantes Joseph SCHELL (1994-1998)

Antiquités sémitiques

Javier TEIXIDOR (1995-2001)

Biologie historique et évolutionnisme Armand DE RICQLÈs (1995-2010)

Philosophie du langage et de la connaissance

Jacques BoUVERESSE (1995-2010)

Norbert OHLER (1994-1995)

Orest RANUM (1994-1995)

1995

Équations différentielles et systèmes dynamiques

Jean-Christophe YocCOZ (1996-2016)

Anthropologie théâtrale

Jerzy GROTOWSKI (1996-1999)

Chimie des solides

Jean RouXEL (1996-1998)

Klaus RAJEWSKI (1995-1996)

Harris MEMEL-Fotê (1995-1996)

1996

Immunologie moléculaire

Philippe KOURILSKY (1998-2012)

Pieter WestbRoeK (1996-1997)

Igor MEL' ‘̌UK (1996-1997)

1997

Histoire de la France des Lumières

Daniel RocHE (1999-2005)

Histoire turque et ottomane

Gilles VEINSTEIN (1999-2012) 
Chaire européenne

Chaire internationale André MiQUEL (1976-1997)

Étude comparée des sociétés africaines Françoise HÉRITIER (1982-1998)

Histoire de la France comtemporaine Maurice AGULHON (1986-1997)

Langues et littératures romanes Harald WEINRICH (1992-1998)

Fondements et principes de la reproduction humaine Étienne-Émile BAULIEU (1993-1998)

Chaire européenne

Chaire internationale
Abram DE SWAAN (1997-1998)

Brian STOCK (1997-1998)

\section{8}

Assyriologie

Jean-Marie DURAND (1999-2011)

Littératures modernes de l'Europe néolatine Carlo Ossola (1999)

Théorie économique et organisation sociale Roger GUESNERIE (2000-2013)

Histoire et civilisation du monde achéménide et de l'empire d'Alexandre Pierre BRIANT (1999-2012)

Biologie et génétique du développement Spyros ARTAVANIS-TSAKONAS (2000-2012)

Thomas W. GAEHTGENS (1998-1999)

Patrice HigONNET (1998-1999)
Analyse mathématique des systèmes et de leur contrôle

Jacques-Louis LIONS (1973-1998)

Histoire de la création artistique en France Jacques THUILLIER (1977-1998)

Grammaire et pensée allemandes Jean-Marie ZEMB (1986-1998)

Histoire économique et monétaire de l'Orient hellénistique

Georges LE RIDER (1993-1998)

Biologie moléculaire des plantes Joseph SCHELL (1994-1998)

Chimie des solides

Jean RouXEL (1996-1998)

Chaire européenne
Théorie des Nombres

Don ZAGIER (2000-2014)

Philosophie des sciences biologiques et médicales

Anne Fagot-Largeault (2000-2009)

Philosophie et histoire des concepts scientifiques

Ian HACKING (2000-2006)

Anthropologie de la nature

Philippe DESCOLA (2000)

Physique quantique

Serge HAROCHE (2001-2015)

Civilisation pharaonique :

Archéologie, philologie, histoire

Nicolas GRIMAL (2000)

Hans-Wilhelm MÜLLER-GÄRTNER (1999-2000) 
Chaire internationale

James Watson CRONIN (1999-2000)
Histoire de la civilisation moderne

Emmanuel LE ROY LADURIE (1973-1999)

Histoire de Rome

Paul VEYNE (1976-1999)

Physique statistique

Philippe NOZIÈRES (1983-2001)

Embryologie cellulaire et moléculaire

Nicole LE DOUARIN (1988-2000)

Anthropologie théâtrale

Jerzy GROTOWSKI (1996-1999)

Chaire européenne

Chaire internationale
Chimie de la matière condensée

Jacques LIVAGE (2001-2009)

Histoire de l'art européen médiéval et moderne

Roland RECHT (2001-2012)

Évolution du climat et de l'océan

Édouard BARD (2001)

Génétique et physiologie cellulaire

Christine PETIT (2001)

Religion, institutions et société de la Rome antique

John SCHEID (2001-2016)

Michael EDWARDS (2000-2001)

Miklós SzABo (2000-2001)
Théorie des groupes

Jacques TITS (1973-2000)

Sociologie

Pierre BOURDIEU (1981-2001)

Antiquités sémitiques

Javier TEIXIDOR (1995-2001)

Chaire européenne

Chaire internationale
Histoire moderne et contemporaine $d u$ politique

Pierre RosAnVALLON (2001)

Études juridiques comparatives et internationalisation du droit

Mireille DELMAS-MARTY (2002-2011)

Équations aux dérivées partielles et applications

Pierre-Louis LIONS (2002)

Claudio MAGRIs (2001-2002)

Paul FARMER (2001-2002)
Histoire et civilisation du monde byzantin Gilbert DAGRON (1975-2001)

Rhétorique et sociétés en Europe (XVI ${ }^{e}$ XVII ${ }^{e}$ siècles) Marc FUMAROLI (1987-2002)
Étude de la création littéraire en langue anglaise

Michael EDWARDS (2003-2008)

Épigraphie et histoire des cités grecques

Denis KNOEPFLER (2003-2014) 
Histoire moderne et contemporaine du monde russe

François-Xavier COQUIN (1993-2001)

Génétique moléculaire

Pierre CHAMBON (1993-2002)

Chaire européenne

Chaire internationale

Pierre JOLIOT (1981-2002)

Chaire européenne

Chaire internationale

Claude COHEN-TANNOUdJI (1973-2004)

Histoire de l'Occident méditerranéen au Moyen-Âge

Pierre TOUBERT (1992-2003)

Chaire européenne

Chaire internationale

Physique de la matière condensée Pierre-Gilles DE GENNES (1971-2004)

Physique corpusculaire

Marcel FROISSART (1973-2004)

Histoire de la France des Lumières Daniel RochE (1999-2005)

Chaire européenne

Chaire internationale

Chaire de création artistique
Histoire contemporaine du monde arabe Henry LAURENS (2003)

Génétique humaine

Jean-Louis MANDEL (2003-2016)

Hans BeLting (2002-2003)

Stuart EDELSTEIN (2002-2003)
Particules élémentaires, gravitation et cosmologie

Gabriele VENEZIANO (2004-2013)

Theodor BERCHEM (2003-2004)

Jayant Vishnu NARLIKAR (2003-2004)

2004

Rationalité et sciences sociales John ELSTER (2006-2011)

Psychologie cognitive expérimentale Stanislas DEHAENE (2005)

Sandro STRINGARI (2004-2005)

Celâl SENGÖR (2004-2005)

\section{5}

Physique mésoscopique

Michel DEVORET (2007-2013)

Littérature française moderne et contemporaine : histoire, critique, théorie Antoine COMPAGNON (2006)

Écrit et cultures dans l'Europe moderne Roger CHARTIER (2006-2016)

Maurice BLOCH (2005-2006)

Thomas PAVEL (2005-2006)

Christian DE PORTZAMPARC (2005-2006) 
Histoire et anthropologie des sociétés méso- et sud-américaines

Nathan WACHTEL (1992-2005)

Chaire européenne

Chaire internationale

Chaire de création artistique

Jean-Pierre CHANGEUX (1975-2006)

Neuropharmacologie

Jacques GLOWINSKI (1982-2006)

Paléoanthropologie et préhistoire

Yves COPPENS (1983-2005)

Théorie linguistique

Claude HAGĖGE (1988-2006)

Les civilisations de l'Europe au néolithique et à l'âge du bronze

Jean GUILAINE (1994-2007)

Philosophie et histoire des concepts scientifiques

Ian HACKING (2000-2006)

Chaire européenne

Chaire internationale

Chaire de création artistique

Chaire Innovation technologique Liliane Bettencourt
Processus morphogénétiques

Alain PROCHIANTZ (2007)

Daniele VITALI (2006-2007)

Guy ORBAN (2006-2007)

Pascal DUSAPIN (2006-2007)
Chimie des processus biologiques

Marc FONTECAVE (2008)

Microbiologie et maladies infectieuses

Philippe SANSONETTI (2007)

Paléoanthropologie humaine

Michel BRUNET (2007-2011)

Milieux bibliques

Thomas RÖMER (2007)

Physique de la matière condensée Antoine GEORGES (2009)

Histoire intellectuelle de la Chine Anne CHENG (2008)

Manfred KROPP (2007-2008)

Pierre MAGISTRETTI (2007-2008)

Ariane MNOUCHKINE (2007-2008), non occupée

Gérard BERRY (2007-2008)

\section{8}

Chaire européenne - Développement durable

Chaire internationale - Savoirs contre pauvreté
Henri LERIDON (2008-2009)

Esther DUFLO (2008-2009) 
Chaire de création artistique

Chaire Innovation technologique Liliane Bettencourt
Pierre-Laurent AIMARD (2008-2009)

Mathias FINK (2008-2009)
Géodynamique

Xavier LE PICHON (1986-2008)

Études de la création littéraire en langue anglaise

Michael EDWARDS (2003-2008)

Chaire de création artistique

Chaire Développement durable Environnement, énergie et société

Chaire Savoirs contre pauvreté

Chaire Informatique et sciences numériques

Chaire Innovation technologique Liliane Bettencourt
Chimie des matériaux hybrides

Clément SANCHEZ (2011)

Métaphysique et philosophie de la connaissance

Claudine TIERCELIN (2010)

Jacques NICHET (2009-2010)

Nicolas STERN (2009-2010)

Peter Piot (2009-2010)

Gérard BERRY (2009-2010)

Patrick COUVREUR (2009-2010)
Histoire des syncrétismes de la fin de l'Antiquité

Michel TARDIEU (1991-2008)

Philosophie des sciences biologiques et médicales

Anne Fagot-Largeault (2000-2009)

Chimie de la matière condensée Jacques LIVAGE (2001-2009)

Chaire de création artistique

Chaire Développement durable Environnement, énergie et société

Chaire Savoirs contre pauvreté

Chaire Informatique et sciences numériques

Chaire Innovation technologique Liliane Bettencourt
Techniques et économies de la Méditerranée antique Jean-Pierre BRUN (2011)

Philologie de la civilisation japonaise Jean-Noël ROBERT (2011)

Physique de l'intérieur de la Terre Barbara ROMANOWICZ (2011)

Anselm KIEFER (2010-2011)

Jean-Marie TARASCON (2010-2011)

Ismail SERAGELDIN (2010-2011)

Martin ABADI (2010-2011)

Elias ZERHOUNI (2010-2011) 
Antiquités nationales

Christian GoudinEAU (1984-2010)

Chimie des interactions moléculaires

Jean-Marie LEHN (1989-2010)

Physiologie de la perception et de l'action Alain BERTHOZ (1992-2010)

Philosophie du langage et de la connaissance

Jacques BOUVERESSE (1995-2010)

Chaire de création artistique

Chaire Développement durable Environnement, énergie et société

Chaire Savoirs contre pauvreté

Chaire Informatique et sciences numériques

Chaire Innovation technologique Liliane Bettencourt

\author{
Atomes et rayonnement \\ Jean DALIBARD (2012) \\ Épigénétique et mémoire cellulaire \\ Edith HEARD (2012) \\ État social et mondialisation \\ Alain SUPIOT (2012) \\ Algorithmes, machines et langages \\ Gérard BERRY (2012)
}

Gilles CLÉMENT (2011-2012)

Paul ColonNA (2011-2012)

Manuela CARNEIRO DA CUNHA (2011-2012)

Serge AвIteBoul (2011-2012)

Jean-Paul LAUMOND (2011-2012)
Histoire du monde indien

Gérard FUSSMAN (1985-2011)

Médecine expérimentale

Pierre CORVOL (1989-2012)

Biologie historique et évolutionnisme Armand DE RICQLÈS (1996-2010)

Assyriologie

Jean-Marie DURAND (1999-2011)

Rationalité et sciences sociales Jon ELSTER (2006-2011)

Paléontologie humaine Michel BRUNET (2007-2011)

Chaire de création artistique

Chaire Développement durable Environnement, énergie et société
Histoire de la philosophie médiévale Alain DE LIBERA (2013)

Médecine expérimentale Alain FISCHER (2013)

Sociologie du travail créateur

Pierre-Michel MENGER (2013)

Histoire et cultures de l'Asie centrale préislamique

Franz GRENET (2013)

Chimie du solide et énergie Jean-Marie TARASCON (2013)

Histoire globale de la première modernité Sanjay SUBRAHMANYAM (2013-2014)

Karol BEFFA (2012-2013)

Annie CAZENAve (2012-2013) 
Chaire Savoirs contre pauvreté

Chaire Informatique et sciences numériques

Chaire Innovation technologique Liliane Bettencourt
Dominique KerouEDAN (2012-2013)

Bernard ChAZELLE (2012-2013)

Yves BRÉCHET (2012-2013)
Immunologie moléculaire

Philippe KOURILSKY (1998-2012)

Histoire et civilisation du monde achéménide et de l'Empire d'Alexandre

Pierre BRIANT (1999-2012)

Études juridiques comparatives et internationalisation du droit

Mireille DELMAS-MARTY (2000-2011)

Biologie et génétique du développement Spyros ARTAVANIS-TSAKONAS (2000-2012)
Histoire du monde indien

Non attribuée

Galaxies et cosmologie

Françoise COMBES (2014)

Civilisation mésopotamienne

Dominique CHARPIN (2014)

Oncologie cellulaire et moléculaire

Hugues DE THÉ (2014)

\section{Nouveaux professeurs invités (chaires pluriannuelles)}

Architecture et forme urbaine

Jean-Louis COHEN (2013-2016)

\section{Chaires annuelles}

Création artistique

Développement durable - Environnement, énergie et société

Savoirs contre pauvreté

Informatique et sciences numériques

Innovation technologique

Liliane Bettencourt
Tony CRAGG (2013-2014)

Gilles BoEUF (2013-2014)

François BouRGUIGNON (2013-2014)

Nicholas AYACHE (2013-2014)

Philippe WALTER (2013-2014)
Immunologie moléculaire

Philippe KOURILSKY (1998-2012)

Histoire turque et ottomane

Gilles VEINSTEIN (1999-2012)

Histoire de l'art européen, médiéval et moderne

Roland RECHT (2001-2012)

Physique mésoscopique

Michel DeVORET (2006-2012)
Culture écrite de l'Antiquité tardive et papyrologie byzantine

Jean-Luc FOURNET (2015)

Histoire du Coran. Texte et transmission François DÉROCHE (2015)

Physique statistique

Bernard DERRIDA (2015)

Économie des institutions, de l'innovation et de la croissance

Philippe AGHION (2015) 


\section{Chaires créées}

Histoire des pouvoirs en Europe occidentale, XIII ${ }^{-} \mathrm{XVI}^{e}$ siècle : Patrick BOUCHERON (2015) Études turques et ottomanes (non attribuée)

\section{Nouveaux professeurs invités (chaires pluriannuelles)}

Paléoanthropologie : Jean-Jacques HubLIN (2014-2017)

Histoire globale de la première modernité : Sanjay SubRAHMANYAM (2014-2019)

\section{Chaires annuelles}

Développement durable - Environnement, énergie et société : Georges CALAS (2014-2015) Informatique et sciences numériques: Marie-Paule CANI (2014-2015)

Innovation technologique Liliane Bettencourt : Bernard MEUNIER (2014-2015)

\section{$2015^{3}$}

\section{Chaires créées}

Géométrie algébrique : Claire VoIsIN (2016)

\section{Chaires annuelles}

Informatique et sciences numériques: Yann LECUN (2015-2016)

Innovation technologique Liliane Bettencourt : José-Alain SAHEL (2015-2016)

Développement durable - Environnement, énergie et société : Thomas STERNER (20152016)

Création artistique : Alain MABANCKOU (2015-2016)

\section{6}

\section{Chaires créées}

Dynamiques du vivant: Thomas LECUIT (2016-)

Religion, histoire et société dans le monde grec antique : Vincianne PIRENNE-DELFORGE (2017-)

Sciences des données: Stéphane MALlat (2017-)

Champs, cordes et gravité : Jean-Marc HeNNEAUX (2017-)

\section{Chaires internationales (pluriannuelles) créées}

Histoire culturelle des patrimoines artistiques en Europe, XVIII ${ }^{e}-X X^{e}$ siècle : Bénédicte SAVOY (2016-2021)

3. À compter du 24 juillet 2014, l'année indiquée est celle de la création de la chaire par le ministre chargé de l'Enseignement supérieur (voir décret $n^{\circ} 2014-838$ du 24 juillet 2014 relatif au Collège de France). Une chaire est fermée lorsque son titulaire n'est plus professeur en activité. Les années indiquées entre parenthèses après le nom des titulaires des chaires sont celles de leur nomination et de leur fin d'activité. 
Architecture et forme urbaine : Jean-Louis COHEN (transformation de l'invitation initiale sur chaire pluriannuelle, 2014-)

Paléoanthropologie: Jean-Jacques HUBLIN (transformation de l'invitation initiale sur chaire pluriannuelle, 2014-)

\section{Chaires annuelles}

Informatique et sciences numériques : Jean-Daniel BoIssonNAT (2016-2017)

Innovation technologique Liliane Bettencourt : Didier Roux (2016-2017)

Création artistique : Philippe MANOURY (2016-2017)

Chaire européenne : Alain WIJFFELS (2016-2017)

\section{7}

\section{Chaires créées}

Migrations et sociétés: François HÉRAN (2017-)

Géographie de l'urbain et du politique : non occupée

Droit, culture et société de la Rome antique : Dario MANTOVANI (2018-)

Sciences du logiciel : Xavier LEROY (2018-)

Matière molle et biophysique : Jean-François JOANNY (2018-)

\section{Chaires internationales (pluriannuelles) créées}

Évolution du génome et développement: Denis Duboule (2017-2022)

Histoire turque et ottomane : Edhem ELDEM (2017-2022)

\section{Chaires annuelles}

Chaire européenne: Victor STOICHITA (2017-2018)

Innovation technologique Liliane Bettencourt: Thomas EBBESEN (2017-2018)

Informatique et sciences numériques : Claire MATHIEU (2017-2018)

\section{8}

Génomique humaine et évolution

Philosophie du langage et de l'esprit

Histoire et archéologie des mondes africains

\section{Chaires annuelles}

Chaire européenne : Lucrezia REICHLIN (2018-2019)

Création artistique : Amos GITAII (2018-2019)

Santé publique: Arnaud FONTANET (2018-2019)

Informatique et sciences numériques : Rachid GUERRAOUI (2018-2019)

Mondes francophones: Yannick LAHENS (2018-2019)

Innovation technologique Liliane Bettencourt: Molly PRZEWORSKI (2018-2019) 